\title{
EPIRETINAL CELL PROLIFERATION \\ IN MACULAR PUCKER AND \\ VITREOMACULAR TRACTION SYNDROME
}

\section{Analysis of Flat-Mounted Internal Limiting Membrane Specimens}

\author{
FEI ZHAO, MS, ARND GANDORFER, MD, CHRISTOS HARITOGLOU, MD, RENATE SCHELER, MTA, \\ MARKUS M. SCHAUMBERGER, PHD, ANSELM KAMPIK, MD, RICARDA G. SCHUMANN, MD
}

\begin{abstract}
Purpose: To describe new details of epiretinal cell proliferation in flat-mounted internal limiting membrane specimens.

Methods: One hundred nineteen internal limiting membrane specimens were removed en bloc with epiretinal membranes from 79 eyes with macular pucker (MP) and 40 eyes with vitreomacular traction syndrome. Intraoperatively, posterior vitreous detachment was assessed as complete or incomplete. Whole specimens were flat-mounted on glass slides and processed for interference and phase-contrast microscopy, cell viability assay, and immunocytochemistry.

Results: Mean cell viability percentage was higher in MP than in vitreomacular traction syndrome. Two cell distribution patterns were found. Anti-CD163 labeling presented predominantly in MP with complete posterior vitreous detachment. CD45 expression was similar in all groups of diagnosis. Anti-glial fibrillary acidic protein (GFAP) labeling was found in MP irrespective of the extent of posterior vitreous detachment. Alpha-SMA ( $\alpha$-smooth muscle actin) labeling was mainly presented in MP with incomplete posterior vitreous detachment and in vitreomacular traction syndrome. Simultaneous antibody labeling included GFAP/CD45, GFAP/CD163, CD163/CD45, and CD163/ $\alpha-S M A$.

Conclusion: Hyalocytes constitute a major cell type of epiretinal cell proliferation in eyes with MP and vitreomacular traction syndrome. Glial cells, notably retinal Muller cells, are involved as well. It appears that transdifferentiation of cells in vitreomacular traction might be more frequent than previously thought and that those cells possess a greater variability of immunocytochemical properties than expected.
\end{abstract}

RETINA 33:77-88, 2013

E piretinal gliosis, such as macular pucker (MP), and vitreomacular traction syndrome (VMTS) are traction vitreoretinopathies that develop at the vitreoretinal interface of the macular area. The major component of the vitreoretinal interface is the internal limiting membrane (ILM) that has attracted enormous interest for

From the Department of Ophthalmology, Ludwig-MaximiliansUniversity, Munich, Germany.

This study is part of a doctoral thesis not published yet.

The authors report no conflicts of interest.

Reprint requests: Ricarda G. Schumann, MD, Department of Ophthalmology, Vitreoretinal and Pathology Unit, LudwigMaximilians-University, Mathildenstrasse 6, 80336 Munich, Germany; e-mail: ricarda.schumann@med.uni-muenchen.de decades. The ILM is the site of epiretinal membrane (ERM) formation, and it mediates tractional forces from the vitreous to retinal layers. There is general consent that vitreoretinal traction plays a crucial role in the pathogenesis and the clinical course of MP and VMTS. ${ }^{1}$ Anteroposterior vitreoretinal traction is caused by persistent vitreoretinal adhesions because of an incomplete posterior vitreous detachment (PVD), whereas tangential traction is caused by contractive ERMs as a result of progressive fibrocellular proliferation at the vitreal side of the ILM. ${ }^{2-6}$

Removal of the vitreous and epiretinal tissue is the principal goal of vitreoretinal surgery in MP and VMTS because ERMs represent the morphological 
correlate of typical symptoms found in these diseases, such as reduction in visual acuity and metamorphopsia. ${ }^{3,7}$ The concept of peeling off the ILM and epiretinal tissue in eyes with traction maculopathies is supported by numerous studies reporting on improved functional and anatomical outcomes. Furthermore, vitreoretinal surgery with ILM/ERM peeling was shown to minimize the recurrence rate of epiretinal cell proliferation. ${ }^{8-11}$

By light and electron microscopic analyses, several studies characterized epiretinal cells and extracellular matrix components. According to their results, ${ }^{11-14}$ various cell types, such as glial cells, hyalocytes, retinal pigment epithelial cells, and fibroblast-like cells, appear to be important in ERM formation. However, the specific role of epiretinal cell proliferation at the ILM in traction maculopathies is still a matter of debate. Even investigations of cell type-specific antigen expressions of epiretinal cells did not allow for identifying the origin of cells exactly, which is most probably because of phenotypic transdifferentiation of epiretinal cells that adopt features of other cell types. To date, there is no specific marker of transdifferentiated cells. ${ }^{15}$

Recently, a new preparation method, the flat-mount preparation of whole ILM specimens, was proposed to overcome limitations of conventional serial sectioning preparation procedures. ${ }^{16-19}$ By processing flatmounted ILM specimens, the whole specimen can be visualized en face, thereby detecting even small single cells on the ILM that would probably not be observed by conventional sectioning procedures. The aim of our study was to obtain more accurate details on epiretinal cell proliferation with regard to cell distribution, cell viability, and cell immunoreactivity using flatmounted ILM/ERM specimens removed en bloc during vitrectomy from eyes with MP and VMTS.

\section{Patients and Methods}

This is a series of 119 surgically excised ILM specimens obtained during vitrectomy for MP and VMTS between January 2008 and April 2010. The specimens consisted of the ILM and epimacular tissue, and they were removed en bloc from 119 eyes of 117 patients including 79 eyes with MP and 40 eyes with VMTS. Intraoperatively, the state of the vitreous was assessed. Regarding the presence of PVD, we divided all specimens from eyes with MP into 2 groups: 1) 39 specimens were removed from eyes with MP and complete PVD; and 2) 40 specimens were removed from eyes with MP and incomplete PVD. Differentiation between MP with incomplete PVD and VMTS was primarily based on the intraoperative assessment of preretinal tissue and vitreomacular traction attachment. Eyes with VMTS presented with firm vitreoretinal adhesions but minor epiretinal cell proliferation, whereas those with MP and incomplete PVD presented with progressive ERMs but minor and slight vitreoretinal adhesions.

All 119 specimens were processed for phasecontrast microscopy, interference microscopy, and cell nuclei staining. LIVE/DEAD cell viability assay was performed in 27 specimens from 27 patients including 10 eyes with MP and complete PVD, 9 eyes with MP and incomplete PVD, and 8 eyes with VMTS (Table 1). For immunocytochemistry, 51 flatmounted ILM specimens from 51 patients were processed, including 16 eyes with MP and complete PVD, 16 eyes with MP and incomplete PVD, and 19 eyes with VMTS. The remaining 40 specimens from 38 patients were only processed for phasecontrast microscopy, interference microscopy, and cell nuclei staining, as mentioned, because these specimens were shown to be multiply folded during flat-mount preparation and were not suitable for immunolabeling procedures.

The records of patients were reviewed for age, gender, previous ocular surgery, and preoperative history such as trauma. Additional information, such as duration of symptoms, was evaluated if available. This study was conducted according to the Declaration of Helsinki and approved by the Institutional Review Board of the Ludwig-Maximlians-University Munich. Informed consent was obtained from all participants involved in this project.

\section{Surgical Procedure and Specimen Collection}

The surgical procedure consisted of a three-port pars plana vitrectomy with en bloc peeling of the ILM and the ERM. All patients were operated by experienced surgeons at the University Eye Hospital Munich. Forty-seven patients underwent a combined procedure of vitrectomy and cataract extraction. Only specimens presenting the ILM were included.

Pars plana vitrectomy was performed as follows. Before opening the infusion line, the status of the posterior hyaloid was determined using a planoconcave contact lens. If the vitreous was partially attached to the retina, PVD was induced by suction with the vitrectomy probe over the optic disk. The posterior hyaloid was detached from the retina and excised up to the periphery. Then, peeling the ILM was performed using an using end-gripping forceps. To improve the precision of ILM peeling and to avoid incomplete removal, vital dyes had been applied during the 
Table 1. Number of Specimens According to Preparation Method

\begin{tabular}{|c|c|c|c|}
\hline $\begin{array}{l}\text { Preparation } \\
\text { Method }\end{array}$ & $\begin{array}{l}\text { MP with } \\
\text { Complete } \\
\text { PVD } \\
(n=39)\end{array}$ & $\begin{array}{c}\text { MP with } \\
\text { Incomplete } \\
\text { PVD } \\
(n=40)\end{array}$ & $\begin{array}{l}\text { VMTS } \\
(\mathrm{n}=40)\end{array}$ \\
\hline $\begin{array}{l}\text { Phase-contrast/ } \\
\text { interference } \\
\text { microscopy }\end{array}$ & 39 & 40 & 40 \\
\hline $\begin{array}{l}\text { Cell staining } \\
\text { by DAPI }\end{array}$ & 39 & 40 & 40 \\
\hline $\begin{array}{l}\text { LIVE/ DEAD } \\
\text { cell viability assay }\end{array}$ & 10 & 9 & 8 \\
\hline $\begin{array}{l}\text { Immunocytochemical } \\
\text { analysis }\end{array}$ & 16 & 16 & 19 \\
\hline
\end{tabular}

surgical procedure: 1) Brilliant blue $\mathrm{G}(0.5 \mathrm{~mL}$, $0.25 \%$, Brilliant Peel; Fluoron $\mathrm{GmbH}$, Neu-Ulm, Germany) was used to stain 20 specimens from MP with complete PVD, 23 specimens from MP with incomplete PVD, and 26 specimens from VMTS; 2) membrane blue $(0.5 \mathrm{~mL}, 0.15 \%$; Dutch Ophthalmic Research Center, Zuidland, the Netherlands) was used to stain 2 specimens from MP with complete PVD and 1 specimen from VMTS; 3) trypan blue $(0.5 \mathrm{~mL}$, 0.15\%; Dutch Ophthalmic Research Center) was used only in 1 specimen from VMTS. Overall, vital dyes were administered in 73 eyes to visualize the ILM. In the remaining 46 eyes, no intravitreal dye was used. In case of coexisting cataract, a combined procedure was performed. The ILM with epimacular tissue was harvested and processed for our study.

\section{Flat-Mount Specimen Preparation}

After removal, the excised tissue was immediately placed into fixative solution of $2 \%$ paraformaldehyde for at least 24 hours, except specimens processed for cell viability testing. Then, specimens were extended onto glass slides as whole mounted membranes to show their maximum area by using fine-tipped forceps under a light microscope (Leica DM2500; Leica, Wetzlar, Germany).

\section{Cell Viability Assay}

The LIVE/DEADViability/Cytotoxicity Kit (Invitrogen, Carlsbad, CA) for mammalian cells constitutes a two-color fluorescence cell viability assay. The polyanionic dye calcein AM is well retained within all cells, producing an intense uniform green fluorescence in all cells. As cell-impermeant viability indicator, ethidium homodimer-1 can produce a bright red fluorescence in dead cells. Using fluorescence microscope in our laboratory, in our study, all cells were stained with blue color by calcein AM, whereas dead cells were shown with red color by binding ethidium homodimer-1. Based on the time of processing specimens after their removal during the surgical procedure, we divided them into 2 groups: 21 specimens were immediately processed after surgical removal; and 6 specimens were processed 24 hours after peeling with meanwhile storage in balanced salt solution (BSS) at $+4^{\circ} \mathrm{C}$.

After preparing ILM specimens as flat-mounted membranes, 1 drop (approximately $100 \mu \mathrm{L}$ ) of LIVE/DEAD reagent was added. Then, specimens were incubated for 30 minutes, and a coverslip was mounted on glass slides. The fluorescence microscope Leica (Leica MS5; Leica) was used for the analysis at magnifications between $\times 50$ and $\times 400$.

\section{Interference and Phase-Contrast Microscopy}

All 119 flat-mounted specimens were examined by interference and phase-contrast microscopy and cell nuclei staining. Cell nuclei staining was performed using 4',6-diamidino-2-phenylindole (DAPI), producing a bight blue color under the fluorescence microscope. We used a modified fluorescence microscope (Leica MS5; Leica) that enabled us to perform both interference and phase-contrast microscopy at magnifications between $\times 50$ and $\times 400$.

\section{Immunocytochemistry}

After fixation, ILM/ERM specimens were rinsed 2 times for 5 minutes with $0.1 \mathrm{M}$ phosphate-buffered saline (pH 7.4) and incubated with the pepsin for 30 minutes at room temperature. Then, specimens were rinsed again 2 times for 5 minutes with $0.1 \mathrm{M}$ phosphate-buffered saline. The samples were blocked using normal donkey serum (dilution, 1:20) in PBTA for 2 hours at room temperature. Afterward, they were rinsed with PBTA 3 times for 5 minutes and then incubated with primary antibodies (anti-CD34, anti-CD45, anti-CD68, anti-CD163, anticollagen IV, anti-CRALBP, anti-pan-cytokeratin, antifibronectin, anti-GFAP, $\alpha$-SMA, anti-GAP-43, anti-Ki 67, anti-Kir4.1, anti-laminin, anti-neurofilament, antivimentin) according to manufacturer's instructions for 24 hours at the incubator $\left(37^{\circ} \mathrm{C}\right)$. Because the maximum number of fluorochromes used at one time was limited, and the antibody combinations were limited because of the species they were originating from, we only used combinations of three antibodies.

The membranes were rinsed for 3 times with PBTA and incubated with the secondary antibodies (cy2, cy3, and cy5) according to manufacturer's instructions for 2 hours at room temperature. Then, specimens were 
rinsed with PBTA 4 times for 10 minutes and with phosphate-buffered saline 3 times for 5 minutes.

Finally, the specimens were prepared as flatmounted membranes. One drop of antifading mounting medium DAPI was added. Then, specimens were placed onto the Crystal Mount (Biomedia, Foster City, CA) with coverslip and viewed under the fluorescence microscope at magnifications between $\times 50$ and $\times 400$.

For control specimens, primary antibodies were substituted with diluent followed by incubation with secondary antibodies alone. All other procedures were identical to immunolabeling procedures mentioned earlier.

\section{Photodocumentation and Statistical Analysis}

Images, captured by a digital camera (ProgRes CF; Jenoptik, Jena, Germany), were analyzed measuring the specimen area in consideration of magnification by Adobe Photoshop CS4. Cell quantification was analyzed using ImageJ software, a Java-based image-processing program developed at the National Institutes of Health. ${ }^{20}$ The dead cell and total cell counts were calculated by ImageJ. Labeled nuclei were counted in fluorescence micrographs using ImageJ software with manual counting. Finally, measured areas and cell counts of specimen parts were added up from each patient.

Statistical analysis of total cell counts and total area of specimens was performed using the computer software, Statistical Package for the Social Science (SPSS, Chicago, IL) version 16.0. Mann-Whitney test, Kruskal-Wallis test, and chi-square test were performed for evaluation. $P<0.05$ was considered to be statistically significant.

\section{Results}

Fifty-nine women and 58 men were included in this series, corresponding to 71 right eyes and 48 left eyes. Two male patients underwent surgery on both eyes. The total of 119 specimens was divided into 3 groups: 1) specimens removed from eyes with MP and complete PVD $(\mathrm{n}=39), 2)$ specimens removed from eyes with MP and incomplete PVD $(n=40)$, and $3)$ specimens removed from eyes with VMTS $(n=40)$.

The average age at the time of surgery was 69.3 years (ranged from 41 to 81 years), and the age distribution of patients was similar in all 3 groups of diagnosis. With regard to previous ocular surgery, 27 eyes had undergone cataract surgery with phacoemulsification and implantation of an intraocular lens, including 6 eyes with MP and complete PVD, 10 eyes with MP and incomplete PVD, and 11 eyes with VMTS.
Among all 119 specimens, 73 samples were removed after administration of vital dyes into the vitreous cavity: Brilliant blue $\mathrm{G}$ was used to stain 20 specimens from MP with complete PVD, 23 specimens from MP with incomplete PVD, and 26 specimens from VMTS; membrane blue was used to stain two specimens from MP with complete PVD and one specimen from VMTS; trypan blue was only used in one specimen from VMTS.

\section{LIVE/DEAD Cell Viability Assay}

LIVE/DEAD assay evaluation focused on a consecutive series of 27 specimens obtained during vitrectomy. In detail, 10 specimens from eyes with MP and complete PVD, 9 specimens from eyes with MP and incomplete PVD, and 8 specimens from eyes with VMTS were tested. The distribution of patients' age and gender involved in this series is shown in Table 2. Figure 1 presents dead cells as red cell nuclei and the amount of total cells as blue cell nuclei.

The viability percentage showed a broad variety from $18 \%$ to $98 \%$ with a mean of $76.6 \%$ (standard deviation [SD]: $27.8 \%$ ). The mean cell viability percentages were 94.5\% (SD: 4.8\%) in specimens removed from eyes with MP and complete PVD, 56.4\% (SD: 28.9\%) in specimens removed from eyes with MP and incomplete PVD, and $64.8 \%$ (SD: 25.9\%) in specimens removed from eyes with VMTS. We found significantly more viable cells in specimens from MP than in specimens from VMTS ( $P=0.012$, Mann-Whitney test). Comparing specimens of MP with complete and incomplete PVD, there was a significant higher viability in specimens from eyes with MP and complete PVD $(P=0.014$, Mann-Whitney test).

Six specimens (including 1 specimen from MP with complete PVD, 3 specimens from MP with incomplete PVD, and 2 specimens from VMTS) were tested 24 hours after surgical removal with meanwhile storage in BSS at $+4^{\circ} \mathrm{C}$. These specimens were found with a mean viability percentage of $83 \%$ (SD: $28 \%$ ), which ranged from $32 \%$ to $98 \%$.

\section{Interference and Phase-Contrast Microscopy}

Total cell count and cell distribution analysis included all 119 specimens. By phase-contrast and interference microscopy, areas of the ILM presenting with cell proliferation were easily distinguished from areas without cell proliferation. The cell nuclei and cell extensions were clearly delineated from the ILM. Fluorescence microscopy showed the cell nuclei with blue color by DAPI stain. 
Table 2. LIVE/ DEAD Viability Assay and Clinical Data

\begin{tabular}{|c|c|c|c|c|c|c|c|c|c|}
\hline Case & Gender & Age & Eye & Diagnosis & Dye & Area $\left(\mathrm{mm}^{2}\right)$ & Total Cells & Live/Dead Cell & Viability (\%) \\
\hline 1 & M & 80 & $\mathrm{R}$ & MP-1 & BBG & 2.9 & 55 & $50 / 5$ & 91 \\
\hline 2 & M & 74 & $\mathrm{R}$ & MP-1 & - & 5.5 & 1719 & $1418 / 3041$ & 82 \\
\hline 3 & $\mathrm{~F}$ & 68 & $\mathrm{R}$ & MP-1 & - & 5.0 & 841 & $810 / 31$ & 96 \\
\hline 4 & M & 72 & $\mathrm{R}$ & MP-1 & - & 3.6 & 1734 & $1686 / 48$ & 97 \\
\hline 5 & $\mathrm{M}$ & 81 & $\mathrm{R}$ & MP-1 & BBG & 2.4 & 663 & 632/31 & 96 \\
\hline 6 & M & 60 & $\mathrm{R}$ & MP-1 & - & 5.4 & 1018 & $906 / 110$ & 89 \\
\hline 7 & $M$ & 71 & $\mathrm{~L}$ & MP-1 & BBG & 16.3 & 6595 & $6298 / 297$ & 95 \\
\hline $8^{*}$ & M & 71 & $\mathrm{R}$ & MP-1 & - & 3.4 & 193 & $185 / 8$ & 96 \\
\hline 9 & $\mathrm{~F}$ & 70 & $\mathrm{R}$ & MP-1 & - & 26.9 & 7459 & $7174 / 285$ & 96 \\
\hline 10 & $\mathrm{~F}$ & 70 & $\mathrm{R}$ & MP-1 & - & 0.9 & 57 & $51 / 6$ & 89 \\
\hline 11 & M & 74 & $\mathrm{R}$ & MP-2 & - & 0.6 & 49 & $35 / 14$ & 71 \\
\hline 12 & $M$ & 83 & $\mathrm{R}$ & MP-2 & - & 16.5 & 6365 & $5992 / 373$ & 94 \\
\hline $13^{*}$ & $\mathrm{~F}$ & 50 & $\mathrm{~L}$ & MP-2 & - & 0.3 & 114 & 99/15 & 87 \\
\hline $14^{\star}$ & $\mathrm{F}$ & 63 & $\mathrm{R}$ & MP-2 & - & 5.8 & 765 & $752 / 13$ & 98 \\
\hline 15 & $M$ & 81 & $\mathrm{~L}$ & MP-2 & BBG & 7.5 & 1391 & 407/984 & 29 \\
\hline 16 & $\mathrm{~F}$ & 44 & $\mathrm{R}$ & MP-2 & BBG & 10.4 & 137 & 109/28 & 80 \\
\hline 17 & $M$ & 62 & $\mathrm{R}$ & MP-2 & - & 8.1 & 780 & $474 / 306$ & 61 \\
\hline 18 & $\mathrm{~F}$ & 66 & $\mathrm{R}$ & MP-2 & - & 23.1 & 7543 & $1876 / 5667$ & 25 \\
\hline $19^{*}$ & $M$ & 45 & $\mathrm{R}$ & MP-2 & - & 14 & 319 & $103 / 216$ & 32 \\
\hline 20 & $\mathrm{~F}$ & 76 & $\mathrm{~L}$ & VMTS & BBG & 6.2 & 231 & 150/81 & 65 \\
\hline 21 & $\mathrm{~F}$ & 76 & L & VMTS & BBG & 3.7 & 14 & $12 / 2$ & 86 \\
\hline 22 & $M$ & 75 & L & VMTS & BBG & 1.1 & 126 & $36 / 90$ & 29 \\
\hline 23 & $\mathrm{~F}$ & 62 & L & VMTS & $\mathrm{BBG}$ & 1.3 & 126 & $46 / 80$ & 37 \\
\hline 24 & $M$ & 65 & $\mathrm{~L}$ & VMTS & BBG & 2.8 & 17 & $3 / 14$ & 18 \\
\hline $25^{\star}$ & $\mathrm{F}$ & 68 & $\mathrm{R}$ & VMTS & - & 2.7 & 446 & $389 / 57$ & 87 \\
\hline $26^{\star}$ & $M$ & 72 & $\mathrm{R}$ & VMTS & - & 1.8 & 11 & $5 / 6$ & 45 \\
\hline 27 & $\mathrm{~F}$ & 74 & $\mathrm{R}$ & VMTS & BBG & 2.6 & 45 & $17 / 28$ & 38 \\
\hline
\end{tabular}

${ }^{*}$ Processed 24 hours after surgical removal.

BBG, brilliant blue G; F, female; M, male; -, no dye; MP-1, MP with complete PVD, MP-2, MP with incomplete PVD.

All 119 specimens showed a large variety of total cell count that ranged from 0 to 20,778 with a mean of 2,550 (SD: 3,930). There was only one specimen found with bare ILM. Cell counts in specimens with homogenous cell distribution were 4,489 (SD: 4,754) compared with 1,298 (SD: 1,168) in specimens with cell clusters. The total area of ILM ranged from $0.3 \mathrm{~mm}^{2}$ to $31.3 \mathrm{~mm}^{2}$ with a mean of $8.8 \mathrm{~mm}^{2}$ (SD: $7.5 \mathrm{~mm}^{2}$ ). Specimens from eyes with MP and complete PVD demonstrated the tendency to present higher cell densities (cell count in relation to area of removed ILM) than those from eyes with MP and incomplete PVD and eyes with VMTS. However, the difference in cell densities was not statistically significant among the 3 groups of diagnosis $(P>0.05$, Kruskal-Wallis test) (Table 3).

Excluding the one specimen removed from MP and complete PVD that presented with bare ILM, cell proliferation was seen as a continuous sheet of cells homogenously distributed on the ILM in half of all 119 cases $(\mathrm{n}=59)$ (Figure 2$)$. In the remaining 59 specimens, cells were inhomogenously distributed at the ILM-forming areas of cell clusters (Figure 3). In detail, cell proliferation was homogenously distributed in $58.9 \%$ (22 of 38 ) specimens removed from MP and complete PVD, in 52.5\% (21 of 40) specimens removed from MP and incomplete PVD, and in $40 \%$ (16 of 40) specimens removed from VMTS.

\section{Immunocytochemical Analysis}

Twenty-seven specimens were processed by indirect immunocytochemical analysis, including 8 specimens from eyes with MP and complete PVD, 8 specimens from eyes with MP and incomplete PVD, and 11 specimens from eyes with VMTS.

Positive immunostaining of anti-GFAP, anti-CD163 and anti-CD45, and anti- $\alpha$-SMA was most predominant in all specimens tested. Detailed analysis of these four antibodies revealed the following: 1) anti-CD163 labeling presented more frequently in MP with complete PVD compared with other groups of diagnosis; 2) $\alpha$-SMA labeling mainly presented more intense in MP with incomplete PVD and VMTS; 3) anti-GFAP labeling was similarly found in both MP groups, irrespectively of the extent of PVD but less frequently in VMTS; and 4) anti-CD45 expressions were similar in all 3 groups of diagnosis.

Most importantly, there were colocalizations of cell type-specific antibodies seen, including anti-GFAP/ 
Fig. 1. LIVE/DEAD viability assay from a specimen removed from a patient with MP and incomplete PVD. A. Dead cells bind to ethidium homodimer-1 presenting as bright red cell nuclei. B. All cells were labeled with calcein AM presenting as blue cell nuclei. Interference micrograph (C) and phase-contrast micrograph (D) of the same detail as (A) and (B) demonstrating the distribution of cells on the ILM (original magnification, $\times 400$ ).
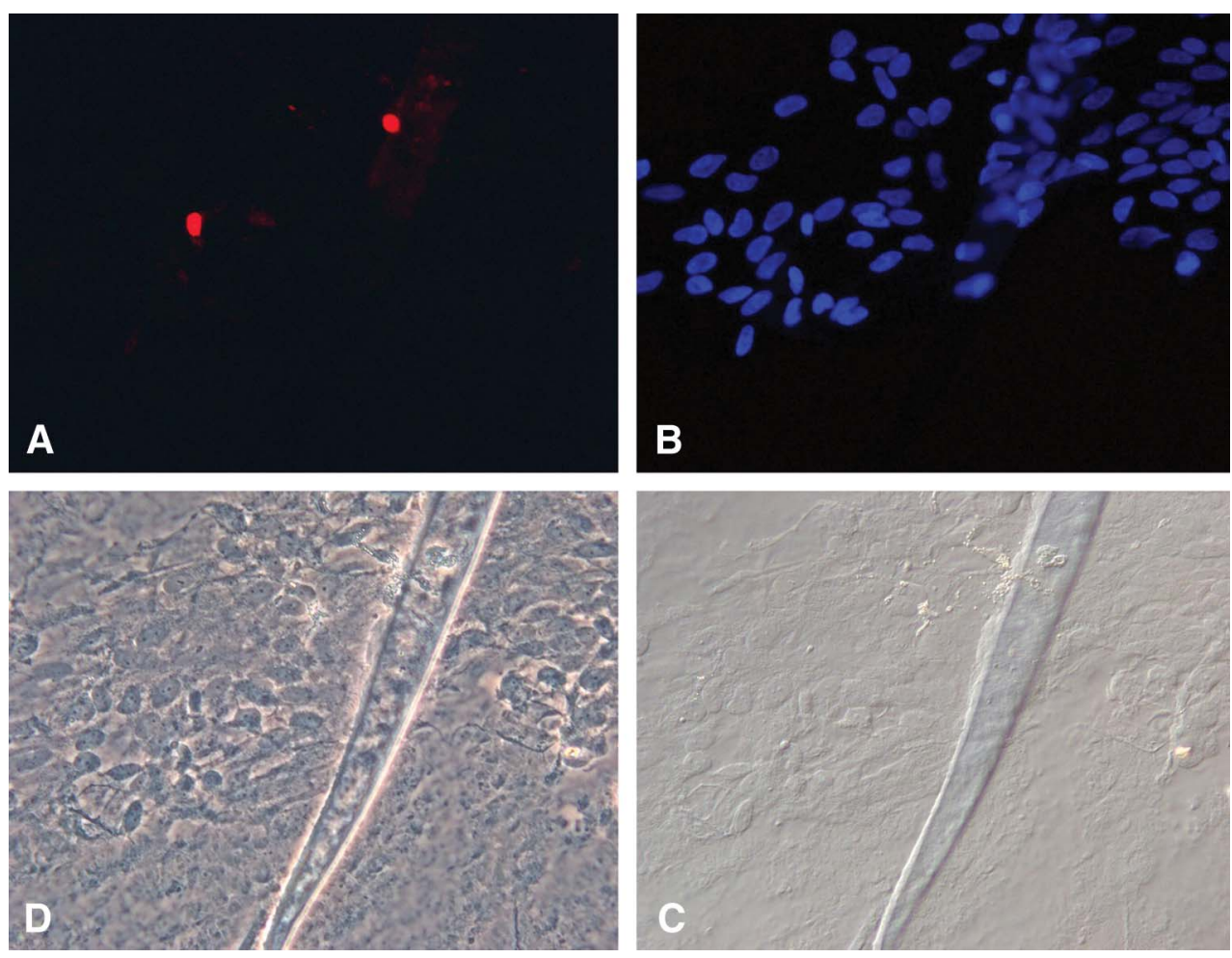

CD45, anti-GFAP/CD163, anti-CD163/CD45, and antiCD163/ $\alpha$-SMA (Figure 4). When labeled with antiGFAP, anti-CD45 was concomitantly colocalized with anti-GFAP. We found colocalization of these 2 antibodies in approximately $20 \%$ of specimens tested. Colocalizations of anti-CD163/GFAP and anti-CD163/CD45 were sparsely present. Colocalization of anti-CD163 and anti- $\alpha$-SMA was only seen in single cases. There was no apparent coexpression of $\alpha$-SMA and GFAP or $\alpha$-SMA and CD45 in any specimen examined. Other cell markers, such as anti-CRALBP and anti-vimentin, were simultaneously expressed in $67 \%$ of tested specimens. However, there was no evidence of colocalization of anti-CRALBP or anti-vimentin with anti-GFAP.

As presented in Table 4 and Figure 5, anti-CRALBP and anti-vimentin were present in all 3 groups of diagnosis (approximately 60\% in each group). Anti-

Table 3. Average Areas of Removed ILM and Total Cell Counts According to the Groups of Diagnosis

\begin{tabular}{lccc}
\hline Diagnosis & $\begin{array}{c}\text { Case } \\
\text { Numbers }\end{array}$ & $\begin{array}{c}\text { Average } \\
\text { Area of } \\
\text { ILM }\left(\mathrm{mm}^{2}\right)\end{array}$ & $\begin{array}{c}\text { Average Total } \\
\text { Cell Count }\end{array}$ \\
\hline $\begin{array}{l}\text { MP with } \\
\text { complete PVD }\end{array}$ & 39 & 9.8 & 3,574 \\
$\begin{array}{l}\text { MP with } \\
\text { incomplete PVD }\end{array}$ & 40 & 9.0 & 2,444 \\
VMTS & 40 & 7.6 & 1,658 \\
\hline
\end{tabular}

Kir4.1 was absent in VMTS specimens but sparsely distributed in specimens from MP. Anti-pan-cytokeratin was only present in one specimen removed for VMTS. Anti-GAP-43 and anti-neurofilament were not seen in this series. Anti-CD34 was sparsely positive in all specimens tested. Anti-CD68 was mostly demonstrated in VMTS (approximately 50\%), was absent in specimens from MP with complete PVD. Collagen IV and fibronectin antigens were expressed in all samples. Anti-laminin was sparsely distributed in MP, irrespectively of the presence of complete or incomplete PVD. In contrast, anti-Ki67 was sparsely distributed in VMTS $(<30 \%)$ but positive in $>60 \%$ specimens removed from eyes with MP.

There were a proportion of cells not labeling with any cell marker combinations used in this study. These unidentified cells were negative for all cell type-specific antibodies used according to Table 4. Negatively labeled cells were mostly found loosely distributed as single cells at the ILM.

\section{Negative Controls}

Whenever more than two parts of specimens from one patient were obtained, we were able to perform indirect immunofluorescence evaluation with more than one combination of three antibodies. In all control specimens, when the primary antibody was substituted by diluent, no immunoreactivity was observed (Figure 6). 

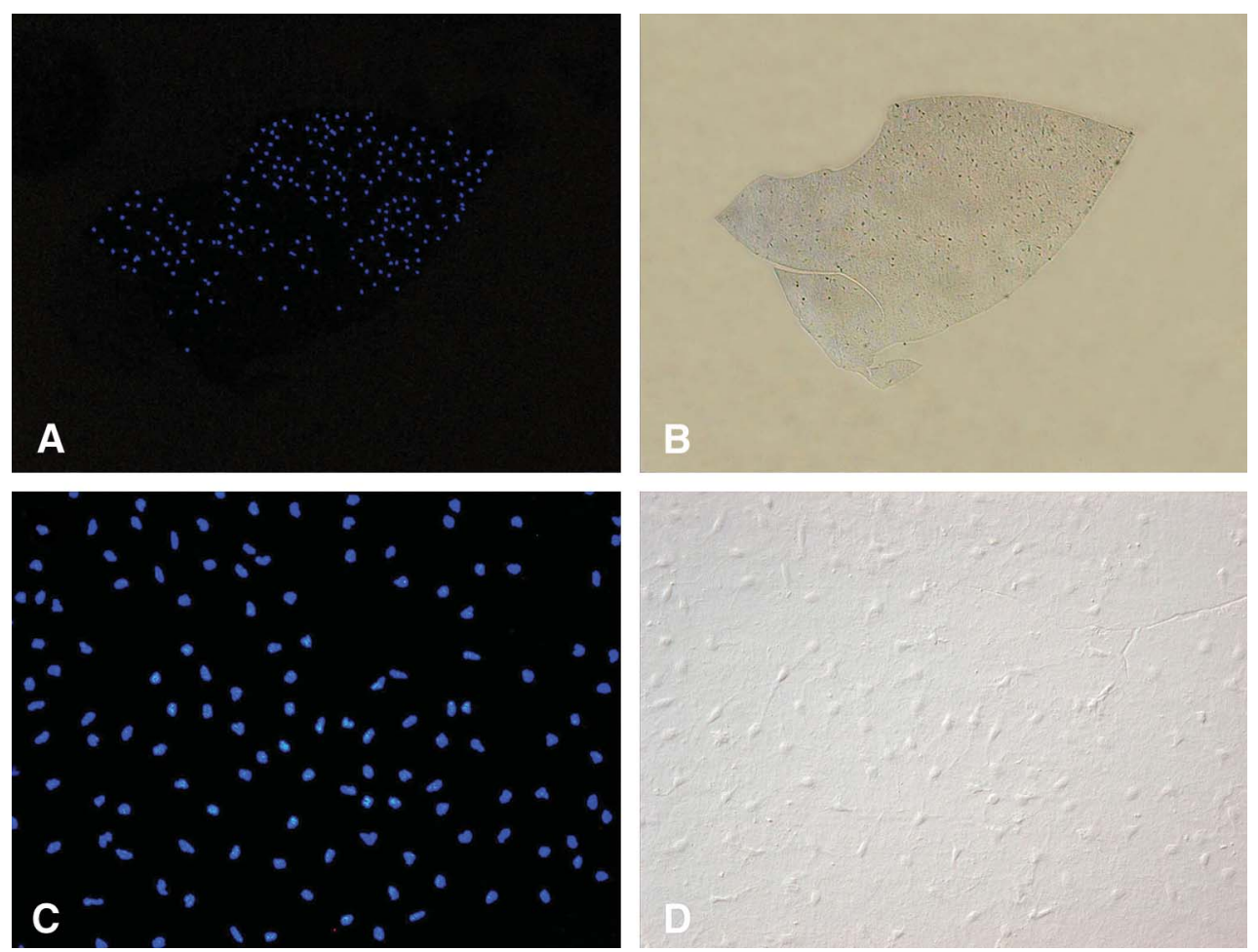

Fig. 2. Homogenous cell distribution patterns in a specimen removed for VMTS. A and C. DAPI cell nuclei staining fluorescence micrograph showing a continuous sheet of homogenously distributed cells on the flat-mounted ILM. Phase-contrast micrograph (B) and interference micrograph (D) presenting the same detail of the specimens (original magnification: $\mathrm{A}$ and $\mathrm{B}$ : $\times 50 ; \mathrm{C}$ and $\mathrm{D}: \times 200$ ).

\section{Discussion}

The new flat-mount preparation method has numerous advantages compared with conventional crosssectioning preparation procedures. Most importantly, it enables to show the maximum area of a tissue specimen with en face observation of the total cell distribution. Furthermore, cell proliferation in flatmounted ILM specimens can be easily examined by different techniques without changing the protocol. However, this procedure does have limitations with regard to morphological topography and the variety of immunocytochemical antibody combinations.

By preparing ILM specimens without fixation as whole flat mounts, we report for the first time on cell viability analysis of ERMs. Significant differences in cell viability of epiretinal cell proliferation in MP and VMTS have been found. Specimens removed from eyes with MP presented with higher cell viability than those removed from eyes with VMTS. In detail, specimens from MP with complete PVD were found with significantly higher cell viability than those from MP with incomplete PVD. These findings raise the hypothesis that the extent of PVD or vitreoretinal adhesion with traction forces may have some influence on cell viability of epiretinal cells, although a causative correlation cannot be determined in this study. Cell viability analysis assesses healthy cells in tissue independently if these cells are actively dividing or if they are quiescent. The fact that ERMs from eyes with complete PVD show significantly more viable cells than those from eyes with persistent vitreoretinal adhesion or vitreomacular traction may be because of an increased cell turnover in the latter ones. One might hypothesize that vitreoretinal traction modulates tissue homeostasis in ERMs, which is a dynamic balance among cell growth, cell proliferation, and cell death to regulate morphology and function. ${ }^{21}$

For cell viability testing, we processed 6 specimens with a time delay of 24 hours after their removal with meanwhile storage in BSS at $+4^{\circ} \mathrm{C}$. These specimens were expected to present lower cell viability percentages than those immediately processed after the surgical procedure. Interestingly, these 6 specimens (1 specimen from MP with complete PVD, 3 specimens from MP with incomplete PVD, and 2 specimens from VMTS) were found with a mean viability percentage of $83 \%$ (SD: $28 \%$ ) that ranged from $32 \%$ to $98 \%$. Because time delay in specimen preparation did not significantly lowered cell viability, we assume that differences in specimens removed from MP and VMTS, as reported above, are rather related to disease characteristics than to tissue processing.

Cell count and cell distribution of ILM/ERM specimens removed from MP and VMTS have not been analyzed before by flat-mount preparation. However, given the fact that we found a large variety of specimen areas in both diseases, total cell numbers remain difficult 
Fig. 3. Inhomogenous cell distribution patterns with cell clusters in a specimen removed for MP with incomplete PVD. $\mathbf{A}$ and C. DAPI cell nuclei staining fluorescence micrographs demonstrating large cell cluster with densely packed cell proliferation (arrow). Phasecontrast micrograph (B) and interference micrograph (D) images showing corresponding details of the same specimens. Cell cluster formation (arrow) sharply delineates directly neighboring bare ILM (asterisk) (original magnification: $\mathrm{A}$ and B: $\times 50$; C and D: $\times 200$ ).
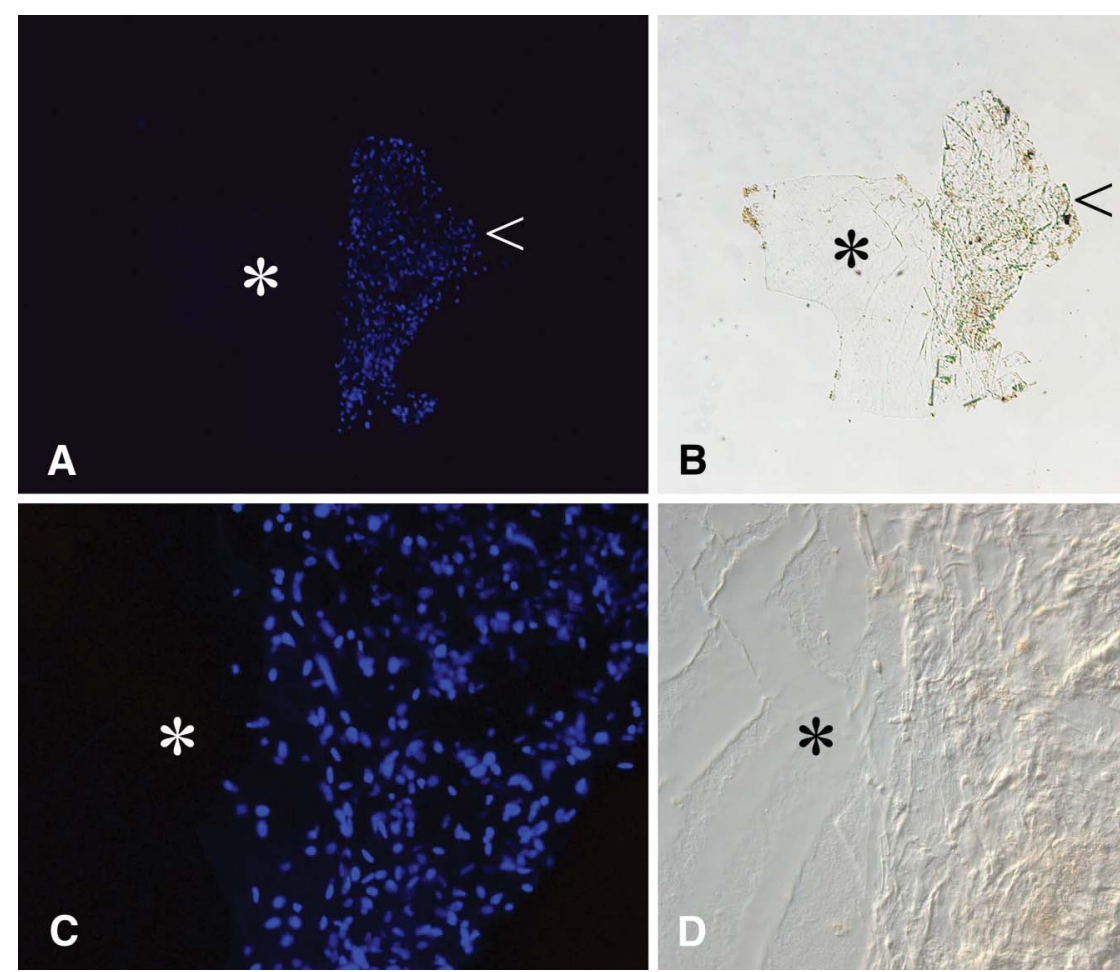

B

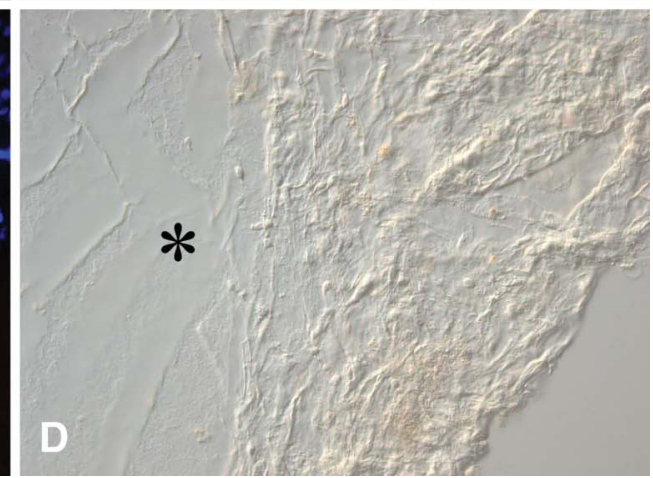

to interpret. Importantly, we found two cell distribution patterns in specimens removed from MP and VMTS: the homogenous cell distribution and the cell cluster formation. Both distribution patterns were reported in flat-mounted ILM specimens removed from eyes with idiopathic macular holes as well. ${ }^{21}$ In this study, specimens from MP demonstrated both distribution patterns, whereas specimens from VMTS presented cell cluster formation more frequently than homogenous cell distribution. On the one hand, one might speculate that this finding supports the hypothesis that epiretinal cell proliferation may be initiated by localized vitreoretinal traction ${ }^{22}$ forming cell clusters that progress after a period to homogenous cell multilayers at the vitreal side of the ILM. Conversely, epiretinal cell proliferation may be initiated by vitreoretinal separation. In eyes with complete PVD, areas of vitreomacular separation would have been present for a longer period than in eyes with partial PVD or VMTS, thereby allowing for cell clusters to progress to homogeneous cell multilayers.

Previously, immunohistochemical examinations were performed by cross-sectional preparation techniques to add information on cell types and the cells' origin involved in ERM formation in MP and VMTS. ${ }^{23-25}$ However, this is the first study reporting on antigen expression in a large series of ILM/ERM specimens that were processed by flat-mount preparation. Here, we present evidence that glial cells and hyalocytes seem to be predominant in epimacular tissue from MP and VMTS.

Glial cell markers, such as GFAP, vimentin, and CRALBP, were demonstrated in specimens from MP and VMTS. Vimentin and CRALBP were seen in MP and VMTS in similar distribution. Kir4.1 was found positive in MP. Immunostaining for pan-cytokeratin was negative in MP and sparsely positive in VMTS. Because 1) retinal Muller glial cells (RMCs) were reported to upregulate GFAP in response to traction forces mediated from the vitreous to the retina, ${ }^{26}$ 2) RMCs are known to be positive for vimentin, ${ }^{27} 3$ ) Kir4.1 was reported to be found on RMC end-feet membranes, ${ }^{28}$ 4) RMCs were demonstrated to be immunoreactive for CRALBP, ${ }^{29}$ and 5) pan-cytokeratin was rather a rare finding in this series, we postulate that rather glial cells than retinal pigment epithelial cells predominated in our specimens. Based on our results, we support the hypothesis that glial cells, notably RMCs, are an important component of ERMs in both MP and VMTS, which is in accordance with previous ultrastructural and immunohistochemical findings. ${ }^{24-26,30}$

Hyalocyte marker, such as CD45 and CD163, were demonstrated similarly in specimens from MP and VMTS in this study. Hyalocytes are known to be immunoreactive for CD45, CD64, and CD163, to belong to the monocyte/macrophage lineage, and to be derived from bone marrow. ${ }^{31-33}$ According to their 

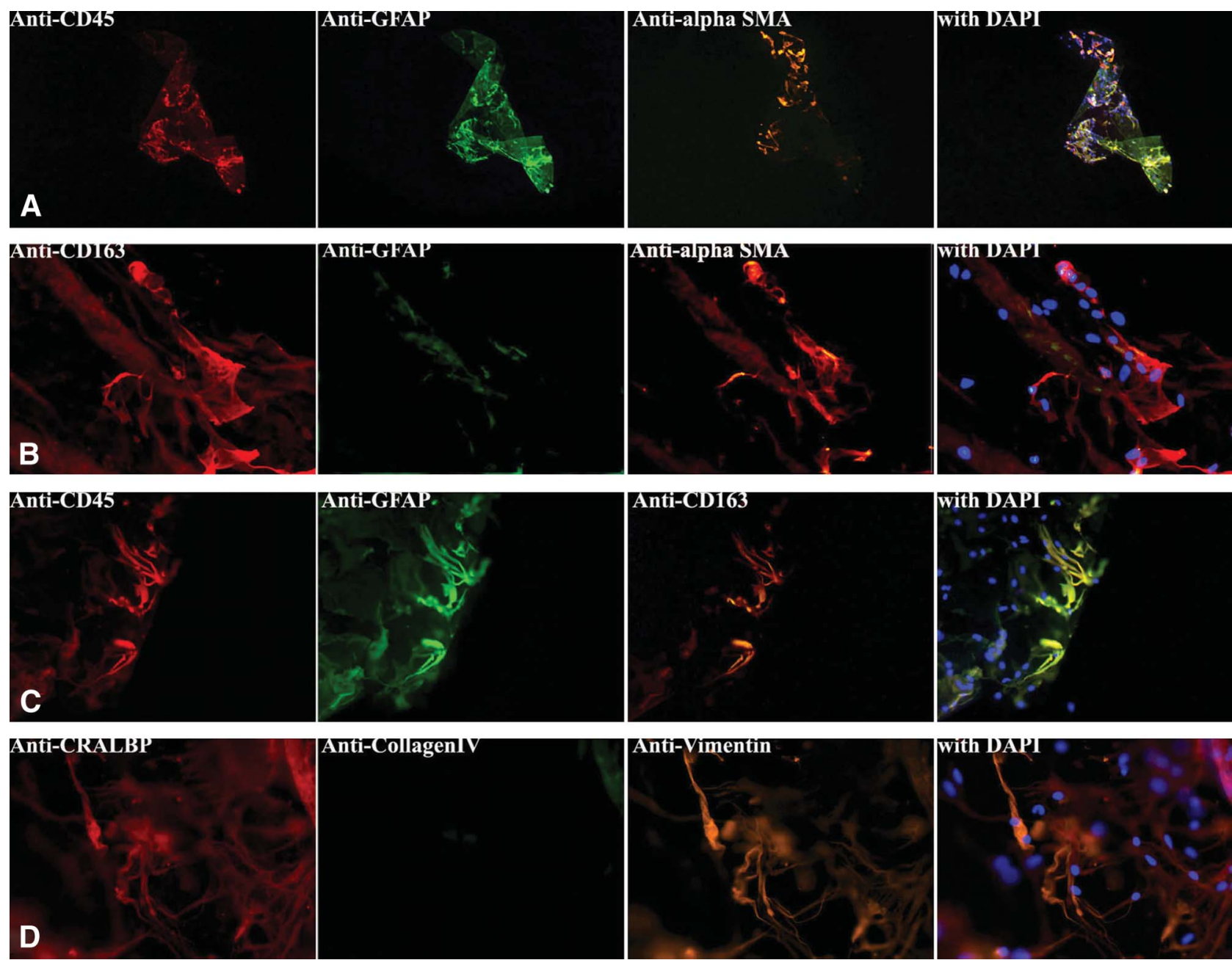

Fig. 4. Fluorescence micrographs demonstrating colocalizations of anti-CD163, anti-CD45, anti-GFAP, anti- $\alpha$-SMA, anti-CRALBP, and antivimentin according to their antibody combinations tested and in combination with a merged figure with DAPI cell nuclei staining. A. Colocalization of anti-GFAP/CD45 with different labeling patterns of anti- $\alpha$-SMA. B. Colocalization of anti-CD163/ $\alpha$-SMA with negative labeling of anti-GFAP. C. Colocalization of anti-GFAP/CD163 and anti-GFAP/CD45. D. Colocalization of anti-CRALBP/vimentin (original magnification: A, $\times 50$; B, $\times 400$; $\mathrm{C}, \times 200 ; \mathrm{D}, \times 400)$.

morphology, hyalocytes are described as resembling macrophages that possess phagocytic activity. However, a variety of morphological features of hyalocytes can be found in cells of the same population. It is unclear if this heterogenicity is related to different origins of cells or to different states of cell metabolism and activity.

Myofibroblast-like cells, being immunoreactive for $\alpha$-SMA, are of unknown origin and represent contractile elements of epiretinal tissue possibly as a consequence of cell transdifferentiation. In this study, $\alpha$-SMA-positive cells were more frequently found in specimens from VMTS and MP with incomplete PVD than in those from MP with complete PVD. They were typically found in ERMs from eyes with VMTS, as reported in previous studies. ${ }^{13,25}$ However, although myofibroblasts are not restricted to membranes derived from VMTS, they can rather be found in all epiretinal tissue that exerts traction at the retina, such as ERMs of eyes from macular holes and idiopathic MP. ${ }^{34,35}$

The cell surface marker CD68 characterizes macrophages and microglia. ${ }^{36}$ In this study, CD68 was demonstrated in VMTS and MP with incomplete PVD. CD68 was not found in specimens from MP with complete PVD. There is no cell-specific marker to differentiate between vitreous-derived macrophages and retinal microglia. With regard to our results, it appears that vitreoretinal traction might be related to the presence of macrophages or microglia in ERMs. CD34 is known to be expressed in endothelial cells of retinal and choroidal blood vessels. ${ }^{37}$ Because antiCD34 was sparsely positive in all specimens tested in this study, we speculate that endothelial cells or endothelial precursor cells do not compose a major component of epiretinal tissue in MP and VMTS. 
Table 4. Antibody Expression According to Diagnosis

\begin{tabular}{lccc}
\hline & \multicolumn{3}{c}{ Positive Labeled Cells Expressed } \\
\cline { 2 - 4 } \multicolumn{1}{c}{ Anti- } & MP with & MP with & \\
& Complete & Incomplete & VMTS \\
PVD $(\mathrm{n}=8)$ & PVD $(\mathrm{n}=8)$ & $(\mathrm{n}=11)$ \\
\hline CD163 & + & + & + \\
CD45 & + & + & + \\
GFAP & + & + & + \\
Vimentin & $(+)$ & + & + \\
CRALBP & + & + & + \\
Kir4.1 & $(+)$ & $(+)$ & - \\
$\alpha$-SMA & + & + & + \\
Pan-Cytokeratin & - & - & $(+)$ \\
GAP43 & - & - & - \\
Neurofilament & - & - & - \\
CD34 & $(+)$ & $(+)$ & $(+)$ \\
CD68 & - & $(+)$ & + \\
Collagen IV & + & + & + \\
Fibronectin & + & + & + \\
Laminin & $(+)$ & $(+)$ & + \\
Ki67 & + & + & $(+)$ \\
\hline
\end{tabular}

-, absent; (+), sparse; + present.

Collagen IV, fibronectin, and laminin was found in all specimens removed from eyes with MP and VMTS, which is in accordance with numerous previous reports. ${ }^{38,39}$ The proliferation marker Ki67 was positive in $>60 \%$ of all tested specimens from eyes with MP in this study, but it was sparsely expressed in VMTS. However, we found no evidence to specify the exact cell types that may be related to the limitation of three antibody combinations. Anti-GAP43 and antineurofilament were not seen in this series, which is in contrast to previous examinations. ${ }^{18,40,41}$

Colocalizations of the glial cell marker (anti-GFAP) and the hyalocyte marker (anti-CD45 and anti-CD163), as demonstrated in this study, have not been described before in human ILM specimens removed for MP and VMTS. Occasionally, we found colocalization of anti$\alpha$-SMA with the hyalocyte marker anti-CD163. Other colocalizations that were observed in this study, such as anti-CD163/CD45 and anti-CRALBP/vimentin, have not been demonstrated in flat-mounted ILM specimens so far, but they are an expected finding according to previous reports on hyalocyte and glial cell antigen expressions. ${ }^{31,32}$ Colocalization of hyalocyte and glial cell marker was recently described in ILM specimens removed for idiopathic macular holes as well. ${ }^{21}$ It appears that GFAP labeling in epiretinal cell proliferation needs to be reconsidered because positive GFAP labeling no longer allows for the determination of cell to be of glial origin. There are two hypotheses that may arise considering our findings. First, cells with double labeling for anti-GFAP and hyalocyte markers may represent hyalocytes. Hyalocytes with positive GFAP expression have already been described in other species. GFAP-positive hyalocytes were reported in porcine, pecteneal, and bovine hyalocyte cell lines. ${ }^{42-44}$ However, it is unknown if these "hyalocytes" expressed GFAP endogenously or

Fig. 5. Immunoreactivity of every antibody used in this study. Except for negative signals in anti-GAP43 and anti-neurofilament labeling, all antibodies demonstrated positive immunoreactivity that was at least sparsely distributed on flatmounted ILM specimens (original magnification: anti-CD163, $\times 200$; anti-CD45, $\times 400$; antiGFAP, $\times 400$; anti-CRALBP, $\times 400$; anti-Kir4.1, $\times 200$; antivimentin, $\times 100$; anti- $\alpha$-SMA, $\times 200$; anti-pan-cytokeratin, $\times 200$; anti-GAP43, $\times 400$; anti-neurofilament, $\times 200$; anti-CD34, $\times 400$; anti-CD68, $\times 400$; anti-collagen IV, $\times 400$; anti-fibronectin, $\times 400$; anti-laminin, $\times 400$; antiKi67, ×400).

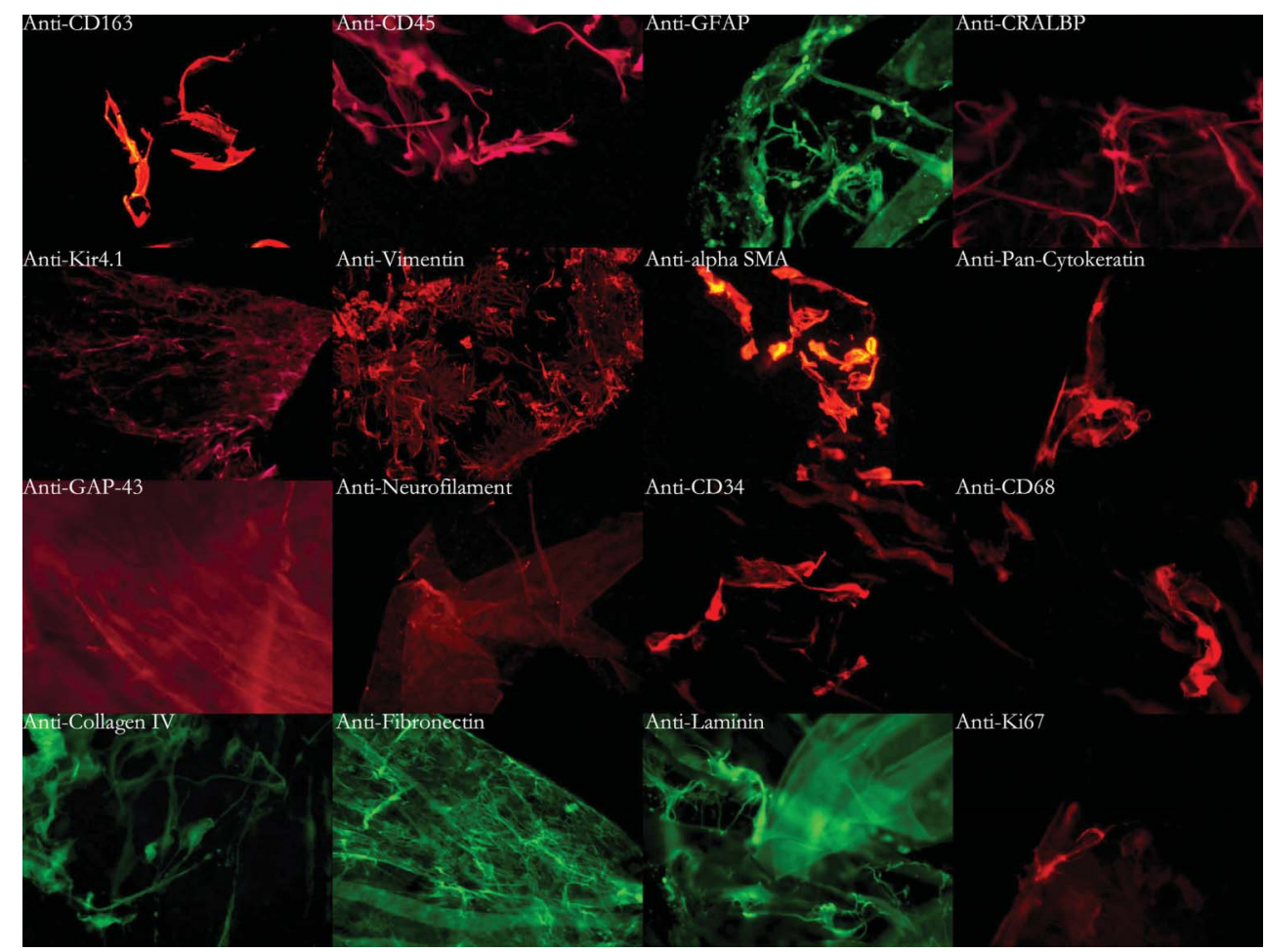




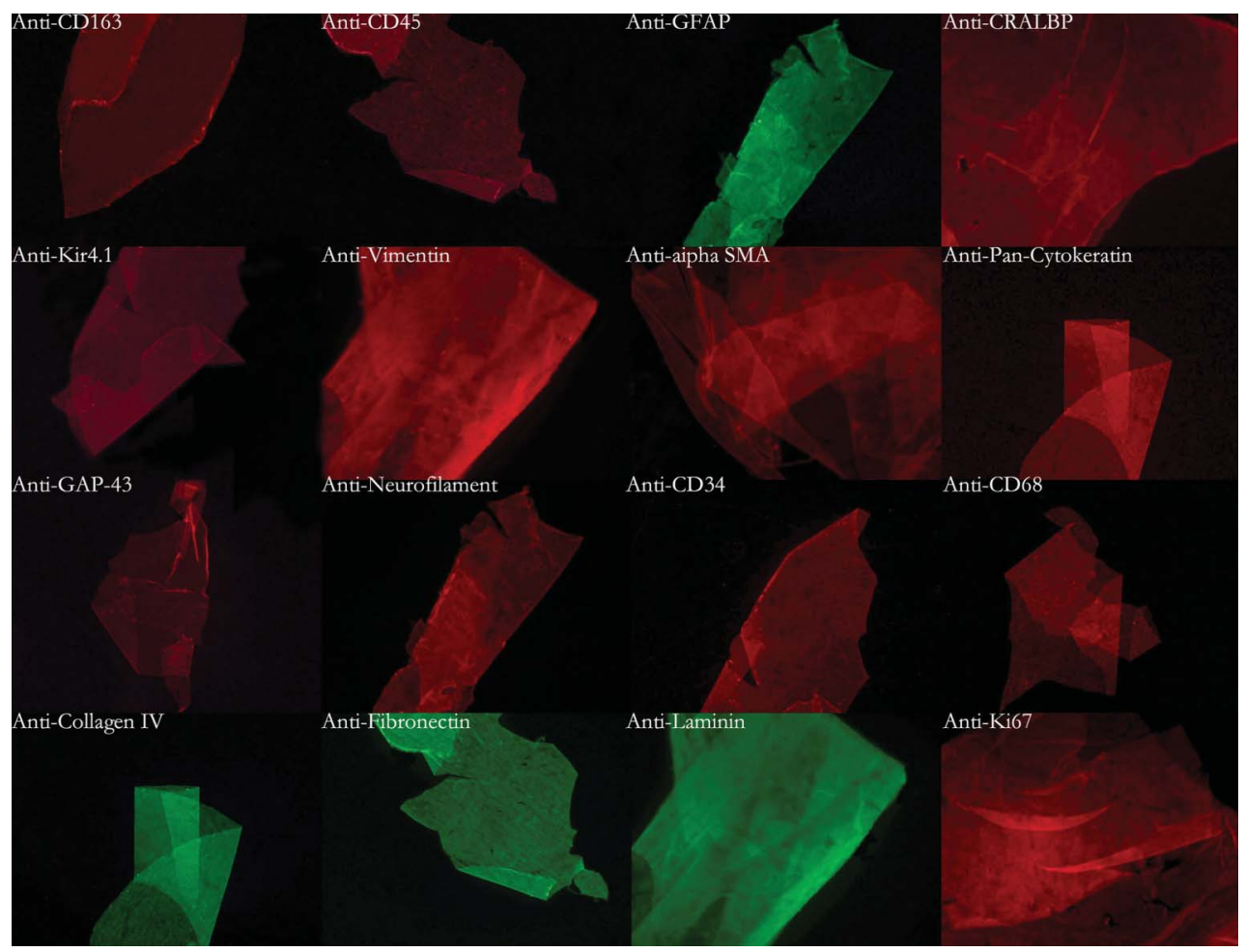

Fig. 6. Fluorescence micrographs of negative controls of immunocytochemical labeling (original magnification: anti-CD163, $\times 50$; anti-CD45, $\times 50$; anti-GFAP, $\times 100$; anti-CRALBP,$\times 400$; anti-Kir4.1, $\times 50$; anti-vimentin, $\times 200$; anti- $\boldsymbol{\alpha}$-SMA, $\times 400$; antipan-cytokeratin, $\times 50$; anti-GAP43, $\times 50$; anti-neurofilament, $\times 100$; anti-CD34, $\times 50$; anti-CD68, $\times 50$; anti-collagen IV, $\times 200$; antifibronectin, $\times 50$; anti-laminin, $\times 50$; anti-Ki67, $\times 200$ )

if they contained GFAP from other origin as a result of their well-known phagocytic activity. Another hypothesis is that double-labeled cells may represent progenitor cells.

Colocalization of anti-CD163/ $\alpha$-SMA indicates that hyalocytes may transdifferentiate into myofibroblastlike cells. Myofibroblast-like transdifferentiation with positive $\alpha$-SMA expression has been shown for both glial cells and hyalocytes. ${ }^{32,44,45}$ Thus, our results support the hypothesis that hyalocytes are able to transdifferentiate into myofibroblast-like cells in ERM formation. Because a proportion of cells were not labeled with any cell marker combination used in this study, we presume that they are 1) cells that are nonviable, or 2) dedifferentiated progenitor cells, or 3) transdifferentiated cells that were not detected by the commonly used immunocytochemical antibodies. Thus, we postulate that transdifferentiation of cells in the vitreous cortex might be more frequent than previously thought and that those cells in the vitreous cortex possess a greater variability of immunocytochemical properties than previously expected. However, it remains to ascertain that these epiretinal cells are capable of exerting traction via cell-mediated contraction of the vitreous cortex in the pathogenesis of vitreomacular traction.

In summary, according to our results, it appears that vitreous-derived cells, namely, hyalocytes, constitute a major cell type of epiretinal cell proliferation in eyes with MP and VMTS. Glial cells, notably RMCs, are involved in ERMs as well. The extent of PVD with persistent vitreoretinal adhesions may play a substantial role in the formation of ERMs. Vitreoretinal traction may be related to the differentiation of epiretinal cells and their antigen expression. Because this study presents rather microscopic observations than causative correlations, further investigation is needed to elucidate the influence of persistent vitreoretinal adhesion on the behavior of epiretinal cell populations.

Key words: cell viability, flat-mount preparation, glial cells, hyalocytes, immunocytochemistry, internal limiting membrane, macular pucker, posterior vitreous detachment, vitreomacular traction.

\section{References}

1. Morris R, Witherspoon CD, Kuhn F, et al. Traction maculopathy. In: Kriegelstein GK, ed. Retinology Today. Munich, Germany: Verlag für Medizin und Naturwissenschaftler; 2000:83-88.

2. Zarbin MA, Michels RG, Green WR. Epiretinal membrane contracture associated with macular prolapse. Am J Ophthalmol 1990;110:610-618.

3. Koerner F, Garweg J. Vitrectomy for macular pucker and vitreomacular traction syndrome. Doc Ophthalmol 1999;97:449-458.

4. Gandorfer A, Rohleder M, Charteris D, et al. Ultrastructure of vitreomacular traction syndrome associated with persistent hyaloid artery. Eye (Lond) 2005;19:333-336.

5. Wylegala E, Woyna-Orlewicz A, Piłat J, et al. Traction maculopathies-pathogenesis and diagnostics. Klin Oczna 2006; 108:457-463. 
6. Sebag J. Vitreochisis. Graefes Arch Clin Exp Ophthalmol 2008;246:329-332.

7. Trese M, Chandler DB, Machemer R. Macular pucker II. Ultrastructure. Graefes Arch Clin Ophthalmol 1983;221:16-26.

8. Park DW, Dugel DU, Garda J, et al. Macular pucker removal with and without internal limiting membrane peeling: pilot study. Ophthalmology 2003;110:62-64.

9. Kwok AK, Lai TY, Li WW, et al. Indocyanine green-assisted internal limiting membrane removal in epiretinal membrane surgery: a clinical and histologic study. Am J Ophthalmol 2004;138:194-199.

10. Kwok Akh, Lai TY, Yuen KS. Epiretinal membrane surgery with or without internal limiting membrane peeling. Clin Experiment Ophthalmol 2005;33:379-385.

11. Tari SR, Vidne-Hay O, Greenstein VC, et al. Functional and structural measurements for the assessment of internal limiting membrane peeling in idiopathic macular pucker. Retina 2007; 27:567-572.

12. Kampik A, Green WR, Michels RG, Nase PK. Ultrastructural features of progressive idiopathic epiretinal membrane removed by vitreous surgery. Am Ophthalmol 1980;90:797-809.

13. Gandorfer A, Rohleder M, Kampik A. Epiretinal pathology of vitreomacular traction syndrome. Br J Ophthalmol 2002;86: 902-909.

14. Sebag J, Gupta P, Rosen RR, et al. Macular holes and macular pucker: the role of vitreoschisis as imaged by optical coherence tomography/scanning laser ophthalmoscopy. Trans Am Ophthalmol Soc 2007;105:121-129; discussion 129-131.

15. Vinores SA, Campochiaro PA, Conway BP. Ultrastructural and electron-immunocytochemical characterization of cells in epiretinal membranes. Invest Ophthalmol Vis Sci 1990;31:14-28.

16. Hisatomi T, Enaida H, Sakamoto T, et al. A new method for comprehensive bird's-eye analysis of the surgically excised internal limiting membrane. Am J Ophthalmol 2005;139:1121-1122.

17. Hisatomi T, Enaida H, Sakamoto T, et al. Cellular migration associated with macular hole: a new method for comprehensive bird's-eye analysis of the internal limiting membrane. Arch Ophthalmol 2006;124:1005-1011.

18. Gandorfer A, Scheler R, Schumann R, et al. Interference microscopy delineates cellular proliferation on flat mounted internal limiting membrane specimens. Br J Ophthalmol 2009;93:120-122.

19. Liu H, Kao WW. A novel protocol of whole mount electroimmunofluorescence staining. Mol Vis 2009;15:505-517.

20. Meth RD, Thompson EB. Hormonal regulation of physiological cell turnover and apoptosis. Cell Tissue Res 2000;301:101-124.

21. Schumann RG, Eibl KH, Zhao F, et al. Immunocytochemical and ultrastructural evidence of glial cells and hyalocytes in internal limiting membrane specimens of idiopathic macular holes. Invest Ophthalmol Vis Sci 2011;3:7822-7834.

22. Bringmann A, Wiedemann P. Involvement of Müller glial cells in epiretinal membrane formation. Graefes Arch Clin Exp Ophthalmol 2009;247:865-883.

23. Hiscott PS, Grierson I, Trombetta CJ, et al. Retinal and epiretinal glia - an immunohistochemical study. Br J Ophthalmol 1984;68:698-707.

24. Heidenkummer HP, Kampik A. Proliferative activity and immunohistochemical cell differentiation in human epiretinal membranes. Ger J Ophthalmol 1992;1:170-175.

25. Shinoda K, Hirakata A, Hida T, et al. Ultrastructural and immunohistochemical findings in five patients with vitreomacular traction syndrome. Retina 2000;20:289-293.

26. Bringmann A, Pannicke T, Grosche J, et al. Müller cells in the healthy and diseased retina. Prog Retin Eye Res 2006;25:397-424.
27. Nakazawa T, Takeda M, Lewis GP, et al. Attenuated glial reactions and photoreceptor degeneration after retinal detachment in mice deficient in glial fibrillary acidic protein and vimentin. Invest Ophthalmol Vis Sci 2007;48: 2760-2768.

28. Higashimori H, Sontheimer H. Role of Kir4.1 channels in growth control of glia. Glia 2007;55:1668-1679.

29. Collery R, McLoughlin S, Vendrell V, et al. Duplication and divergence of zebrafish CRALBP genes uncovers novel role for RPE- and Muller-CRALBP in cone vision. Invest Ophthalmol Vis Sci 2008;49:3812-3820.

30. Gastaud P, Bétis F, Rouhette H, Hofman P. Ultrastructural findings of epimacular membrane and detached posterior hyaloid in vitreomacular traction syndrome. J Fr Ophthalmol 2000;23:587-593.

31. Lazarus HS, Hageman GS. In situ characterization of the human hyalocytes. Arch Ophthalmol 1994;112:1356-1362.

32. Sakamoto T, Ishibashi T. Hyalocytes: essential cells of the vitreous cavity in vitreoretinal pathophysiology? Retina 2011;31:222-228.

33. Qiao H, Hisatomi T, Sonoda KH, et al. The characterisation of hyalocytes: the origin, phenotype, and turnover. Br J Ophthalmol 2005;89:513-517.

34. Smiddy WE, Michels RG, Gilbert HD, Green WR. Clinicopathologic study of idiopathic macular pucker in children and young adults. Retina 1992;12:232-236.

35. Schumann RG, Rohleder M, Schaumberger MM, et al. Idiopathic macular holes: ultrastructural aspects of surgical failure. Retina 2008;28:340-349.

36. Streit WJ. Microglia and macrophages in the developing CNS. Neurotoxicology 2001;22:619-624.

37. Barcelona PF, Luna JD, Chiabrando GA, et al. Immunohistochemical localization of low density lipoprotein receptor-related protein 1 and alpha(2)-macroglobulin in retinal and choroidal tissue of proliferative retinopathies. Exp Eye Res 2010;91: 264-272.

38. Chen W, Mo W, Sun K, et al. Microplasmin degrades fibronectin and laminin at vitreoretinal interface and outer retina during enzymatic vitrectomy. Curr Eye Res 2009;34: $1057-1064$.

39. Ponsioen TL, van Luyn MJ, van der Worp RJ, et al. Collagen distribution in the human vitreoretinal interface. Invest Ophthalmol Vis Sci 2008;49:4089-4095.

40. Lesnik Oberstein SY, Lewis GP, Dutra T, Fisher SK. Evidence that neurites in human epiretinal membranes express melanopsin, calretinin, rod opsin and neurofilament protein. $\mathrm{Br} \mathrm{J}$ Ophthalmol 2011;95:266-272.

41. Dijk F, Bergen AA, Kamphuis W. GAP-43 expression is upregulated in retinal ganglion cells after ischemia/reperfusioninduced damage. Exp Eye Res 2007;84:858-867.

42. Llombart C, Nacher V, Ramos D, et al. Morphological characterization of pecteneal hyalocytes in the developing quail retina. J Anat 2009;215:280-291.

43. Nishitsuka K, Kashiwagi Y, Tojo N, et al. Hyaluronan production regulation from porcine hyalocyte cell line by cytokines. Exp Eye Res 2007;85:539-545.

44. Kohno RI, Hata Y, Kawahara S, et al. Possible contribution of hyalocytes to idiopathic epiretinal membrane formation and its contraction. Br J Ophthalmol 2009;93:1020-1026.

45. Hirayama K, Hata Y, Noda Y, et al. The involvement of the rho-kinase pathway and its regulation in cytokine-induced collagen gel contraction by hyalocytes. Invest Ophthalmol Vis Sci 2004;45:3896-3903. 